\section{A interiorização da epidemia de HIV/AIDS e o fluxo intermunicipal de internação hospitalar na Zona da Mata, Minas Gerais, Brasil: uma análise espacial}

\author{
Decentralization of the HIV/AIDS epidemic and \\ inter-municipal flow of hospital admissions in the \\ Zona da Mata, Minas Gerais State, Brazil: \\ a spatial analysis
}

\author{
Cláudia Tartaglia Reis 1 \\ Dina Czeresnia 2 \\ Christovam Barcellos 3 \\ Wagner Souza Tassinari 2,4
}

\footnotetext{
I Secretaria Municipal de Saúde de Cataguases, Cataguases, Brasil.

2 Escola Nacional de Saúde Pública Sergio Arouca,

Fundação Oswaldo Cruz, Rio de Janeiro, Brasil. 3 Centro de Informação Científica e Tecnológica Fundação Oswaldo Cruz, Rio de Janeiro, Brasil.

4 Universidade Federal Rural do Rio de Janeiro, Seropédica, Brasil.

Correspondência C. T. Reis

Secretaria Municipal de Saúde de Cataguases. Av. Astolfo Dutra 600, apto. 603, Cataguases, MG 36770-001, Brasil. clautartaglia@gmail.com
}

\section{Abstract}

The aim of this article was to analyze the decentralization of the HIVIAIDS epidemic (a shift towards rural areas or away from the coast) and to investigate access to HIVIAIDS services from 1988 to 2002 in the Zona da Mata, Minas Gerais State, Brazil. An ecological study was performed using temporal and spatial approaches. A hospital admissions flow between municipalities developed, and the reference group was AIDS patients over 15 years of age admitted in 1996 and 2004, residing in municipalities in the Zona da Mata. There were 2,469 reported AIDS cases in individuals over 15 during the period. Mean incidence and mortality rates were calculated and recalculated by a local empirical Bayesian method in order to more clearly represent the municipalities with the highest concentration of cases and deaths. Decentralization of the epidemic was observed. Juiz de Fora was the municipality with the most cases and may have acted as a hub for spread of HIV in the region. Hospital care for AIDS cases in the Zona da Mata was concentrated in that municipality. There is a strong need to further investigate why referral hospitals in other municipalities in the region have not participated in providing management and care for HIVIAIDS patients.

HIV; Acquired Immunodeficiency Syndrome; Hospitalization; Spatial Analysis

\section{Introdução}

A AIDS no Brasil evoluiu de forma diferenciada e seu perfil epidemiológico sofreu muitas transformações desde o registro dos primeiros casos, no início da década de 1980. A epidemia foi progressivamente disseminada entre mulheres, por meio de relações heterossexuais sem proteção, caracterizando um processo de feminização e heterossexualização 1,2,3,4. Outra tendência foi a síndrome acometer pessoas com menor nível de escolaridade, o que foi denominado processo de pauperização $2,4,5$. Numa ampla perspectiva social e geográfica, observou-se também a chamada interiorização, ou seja, a propagação da epidemia para um número cada vez maior de municípios distantes das principais áreas metropolitanas, atingindo fortemente aqueles que vivem em comunidades menos assistidas 2,3,4,6,7.

Embora o número de casos absolutos de AIDS seja maior nas duas cidades brasileiras mais populosas, São Paulo e Rio de Janeiro, as taxas de incidência revelam importante disseminação geográfica da epidemia. No ano 2000, das dez cidades com maior taxa de incidência, apenas duas delas eram capitais de estado (Porto Alegre em 3o e Florianópolis em 4o lugar), estando São Paulo e Rio de Janeiro em 32o e 34으, respectivamente 8 .

No Estado de Minas Gerais, embora também ocorra uma concentração de casos nas grandes cidades, existem registros de casos em 580 dos 853 municípios 9 . Proietti \& Caiaffa 10 realizaram 
estudo sobre a situação e tendências da epidemia de AIDS em Minas Gerais, no período de 1983 a 1995, tendo como unidade de análise as regiões administrativas do estado, e constataram que a epidemia encontrava-se em diferentes níveis de maturação, dependendo da região geográfica. Nesse estudo, a Zona da Mata apresentava-se entre as quatro regiões com maior número de casos no estado, com a epidemia bem estabelecida a partir de 1987.

A difusão da epidemia em populações cada vez mais heterogêneas foi paralela aos avanços tecnológicos alcançados no tratamento do HIV/ AIDS. Especialmente a distribuição gratuita da terapia anti-retroviral de alta potência (Hight Active Antiretroviral Terapy - HAART) pelo Ministério da Saúde, a partir de 1997, a qual se traduziu em expressiva melhoria na sobrevida com a queda da mortalidade em cerca de $50 \%$ e redução das infecções oportunistas 11 .

As diretrizes da municipalização e regionalização dos serviços de saúde, fundamentadas na Norma Operacional Básica do Sistema Único de Saúde de 1996 (NOB-SUS/96) e nas Normas Operacionais de Assistência à Saúde de 2001 e 2002 (NOAS-2001/2002), respectivamente, incentivaram a organização e articulação dos serviços em nível local, buscando atender aos princípios básicos do sistema, de universalidade, integralidade e eqüidade. Esses serviços estruturam-se por níveis de atenção, que variam segundo suas densidades tecnológicas. No entanto, no que concerne à organização dos serviços de prevenção e atenção à saúde do portador de HIV/ AIDS, a viabilização dessas diretrizes é complexa: o grande desafio reside na provisão eqüitativa de tratamentos para a AIDS, necessária para garantir aumento na sobrevida em todos os segmentos da população infectada pelo HIV.

O paciente em tratamento anti-retroviral é monitorado por uma grade anual de consultas e exames, revisada periodicamente pelo Consenso Nacional. O acesso universal à terapia torna-se factível apenas em um contexto de integralidade das ações em HIV/AIDS. A prevenção, aconselhamento e testagem, e altos níveis de adesão ao tratamento são essenciais para sustentação da universalidade do acesso 12 .

A centralização de unidades prestadoras de ações e serviços em HIV/AIDS confronta-se com a expansão geográfica da epidemia, traduzida pelo processo de interiorização. Os serviços de atendimento especializados, modalidade ambulatorial criada em 1993 pelo Ministério da Saúde, ainda que não exijam, necessariamente, tecnologia sofisticada, concentram-se nos grandes centros urbanos. Tais serviços contemplam o trabalho multiprofissional como condição mínima pa- ra atendimento aos portadores de HIV/AIDS 12, viabilizando a integralidade da assistência, fato que inclui a dispensação de medicamentos antiretrovirais. Os pacientes residentes em municípios de pequeno porte precisam se deslocar para serviços de referência do SUS em centros urbanos de maior porte.

As maiores dificuldades de acesso ao tratamento dos doentes residentes em regiões mais distantes dos grandes centros urbanos constituem um desafio para a universalização dos avanços terapêuticos alcançados na luta contra a epidemia. Nesse sentido, viver em um município menor e mais distante dos lugares que concentram recursos assistenciais é um aspecto que acrescenta maior vulnerabilidade ao doente de AIDS.

Em Minas Gerais, a organização dos serviços de saúde é estabelecida pelo Plano Diretor de Regionalização (PDR) ${ }^{13}$. A região da Zona da Mata abrange duas macrorregiões de saúde, que somam juntas 11 microrregiões e 146 municípios. Desses, aproximadamente $57 \%$ possuem população inferior a 5 mil habitantes.

A assistência ambulatorial ao paciente portador de HIV/AIDS, incluindo a dispensação de anti-retrovirais, é referenciada e centralizada no Município de Juiz de Fora. Para atender às demandas de hospitalização por HIV/AIDS na região, há cinco unidades hospitalares cadastradas: dois hospitais localizados em Juiz de Fora, município pólo macrorregional, e outros três situados em Leopoldina, Ponte Nova e Muriaé, municípios-pólo microrregionais 14 .

Com o objetivo de aprofundar a compreensão acerca desse problema, este artigo analisa o processo de interiorização da epidemia na Zona da Mata de Minas Gerais, relacionando-o com a questão do fluxo dos serviços de assistência aos portadores do HIV/AIDS.

\section{Materiais e métodos}

Foi realizado um estudo ecológico, utilizando a análise espacial como técnica de tratamento dos dados e o município como unidade geográfica de análise.

O processo de interiorização da AIDS compreende diversos aspectos, dentre eles a tendência de espalhamento da epidemia, dos maiores centros urbanos, em geral localizados ao longo do litoral, para outras regiões. Outra conseqüência é o acometimento de indivíduos residentes em municípios de pequeno porte (como é o caso da região do estudo, onde cerca de $57 \%$ dos municípios possuem menos de 5 mil habitantes), o que acarreta em problemas metodológicos para 
a análise de tendências. Devido à instabilidade dos indicadores, gerada pelos pequenos números de população e de eventos (casos e óbitos por AIDS), duas estratégias complementares foram adotadas nesse trabalho: a suavização das variações desses indicadores levando em consideração a estrutura espacial de dependência e a agregação dos indicadores em largos períodos de tempo.

Os critérios de periodização do estudo foram os seguintes: a mudança de base cartográfica de referência que interfere no cálculo de indicadores - optou-se por cortes em anos eleitorais, que coincidem com épocas de emancipação de municípios; o advento da HAART, especialmente a partir de 1997, que impactou mudanças na dinâmica da epidemia; e a descentralização dos serviços de saúde, relacionada ao acesso e à eqüidade dos serviços de saúde. Fixou-se a periodização do estudo da seguinte forma: (a) 1988 a 1992 - epidemia predominantemente masculina, localizada nas grandes metrópoles; (b) 1993 a 1996 - franco processo de feminização, heterossexualização e interiorização da epidemia; (c) 1997 a 2002 - universalização da HAART e descentralização dos serviços de saúde.

O período de 2003 a 2004 foi desprezado devido a problemas de atraso de notificação no SINAN/AIDS (Sistema de Agravos de Notificação de AIDS) 15 .

Neste estudo, a Zona da Mata foi circunscrita aos 146 municípios das macrorregiões de saúde sudeste e leste do sul do Estado de Minas Gerais, definidas pelo PDR do Estado13.

Na primeira parte do estudo - a investigação da interiorização da epidemia -, o evento marcador foi constituído pelos casos notificados por AIDS, maiores de 15 anos, no período compreendido entre 1988 e 2002, residentes nos municípios da Zona da Mata. Foram estimadas as taxas médias de incidência e mortalidade por HIV/AIDS dos municípios, nos três períodos do estudo.

A taxa bruta é o estimador mais simples para medir o risco de ocorrência de um evento, definindo-se como a razão entre o número de eventos ocorridos na área e o número de pessoas expostas à ocorrência desse evento. Contudo, um grande problema associado ao uso dessas taxas é a alta instabilidade que elas possuem quando o evento é raro e a população da região de ocorrência é pequena. As variações bruscas que ocorrem com essas taxas podem nada ter a ver com o fenômeno e sim com uma variabilidade associada às observações. Flutuações aleatórias casuais como a ocorrência de um ou dois eventos a mais ou a menos numa localidade com população pequena causam variações substanciais nas taxas brutas. Além disso, para situações em que não ocorrem casos do evento, a taxa bruta estima o risco de ocorrência do evento como zero, algo irreal em se tratando de dados como doenças ou crimes 16 .

As taxas médias de incidência e mortalidade por HIV/AIDS foram reestimadas pela utilização do método bayesiano empírico local. A metodologia bayesiana espacial estima taxas corrigidas com base nos valores observados, utilizando-se conceitos de inferência. O estimador Bayes Empírico Local inclui efeitos espaciais, calculando a taxa localmente, usando somente os vizinhos geográficos da área na qual se deseja estimar a taxa, convergindo em direção a uma média local. As taxas corrigidas são menos instáveis, pois levam em conta no seu cálculo não só a informação da área, mas também a informação da sua vizinhança. Se a localidade apresenta uma população considerável, a taxa apresentará pequena variabilidade e permanecerá praticamente inalterada quando comparada à taxa bruta. Por outro lado, se a localidade apresentar uma população pequena, a estimativa da taxa bruta terá grande variância e pouco peso será atribuído a essa taxa não estável, tornando a taxa bayesiana mais próxima do valor esperado de uma área escolhida ao acaso naquela região 16,17. Mapas baseados nessas estimativas apresentam, por isso, tendências globais de difusão da epidemia. Os mapas construídos com essas taxas brutas apresentam a ocorrência observada de eventos, a qual pode ter um maior espalhamento, refletindo processos de difusão não necessariamente contínuos no espaço.

Na segunda parte do estudo - a análise do acesso aos serviços em HIV/AIDS -, a proposta inicial era trabalhar dados ambulatoriais que expressassem o acesso do paciente ao serviço de saúde. Na assistência ambulatorial, o paciente recebe os medicamentos anti-retrovirais e acompanhamento para a adesão à terapia. No entanto, o Sistema de Informações Ambulatoriais do SUS (SIA-SUS) apresenta problemas de qualidade e consistência, situação que inviabilizou a sua utilização para este fim. Optou-se, portanto, pela construção do fluxo intermunicipal de internação hospitalar, para fins de investigar o acesso dos pacientes aos serviços de assistência. Construiu-se o fluxo de internação para os anos de 1996 e 2004, tendo como referência os pacientes maiores de 15 anos internados pelo SUS, residentes nos municípios da Zona da Mata. O critério de escolha para construção dos fluxos nos anos de 1996 e 2004 foi o advento da HAART, cuja dispensação se efetivou na rede pública a partir de 1997. Logo, esperavam-se padrões distintos entre as internações ocorridas em 1996 (antes do acesso à HAART) e 2004 (sete anos após o aces- 
so à terapia). A identificação das internações por AIDS no Sistema de Internação Hospitalar (SIHSUS) se deu mediante o diagnóstico principal ou secundário. Foi utilizada a 9a Revisão da Classificação Internacional de Doenças (CID-9) 18 para o ano de 1996 e a 10a Revisão da CID 19, para o ano de 2004. A construção dos fluxos foi realizada por intermédio da confecção de uma tabela de origem (município de residência do paciente) e destino (município de internação), geradas pelo TABWIN (Departamento de Informática do SUS. http://portal.saude.gov.br/portal/se/datasus/ area.cfm?id_area=732).

Foram utilizados como fontes de dados: o SINAN-AIDS da Secretaria de Estado da Saúde de Minas Gerais e o Sistema de Informação sobre Mortalidade (SIM), do Ministério da Saúde, para a investigação da interiorização da epidemia; o SIH-SUS do Ministério da Saúde foi utilizado na construção do fluxo intermunicipal de internação; os Censos Demográficos de 1991 e 2000 do Instituto Brasileiro de Geografia eEstatística (http: //www.ibge.gov.br) foram utilizados na construção das Taxas de Incidência e Mortalidade por HIV/AIDS.

A integração dos programas TABWIN e R (The R Foundation for Statistical Computing, Viena, Áustria; http:/ / www.r-project.org) e sua interface com o TerraView (Instituto Nacional de Pesquisas Espaciais; http://www.dpi.inpe.br/terraview), todos programas livres utilizados neste estudo, permitiram ampliar as análises dos dados fornecidos pelo Ministério da Saúde, por meio do DATASUS. A integração entre esses pacotes estatísticos e de análise espacial foi realizada por meio de arquivos que são compatíveis com um grande número de pacotes comerciais e livres (dbf para dados e shp para mapas).

O estudo envolveu a coleta e análise documental de dados secundários, tendo sido aprovado pelo Comitê de Ética em pesquisa da Escola Nacional de Saúde Pública Sergio Arouca, Fundação Oswaldo Cruz.

\section{Resultados}

Foram notificados 2469 casos de AIDS em maiores de 15 anos na área de abrangência e período do estudo. Dentre eles, $68,13 \%$ eram residentes no Município de Juiz de Fora.

Foram calculadas as taxas médias de incidência para os três períodos do estudo. A interiorização da epidemia pôde ser visualizada ao longo dos períodos por um padrão irregular, mostrando taxas altas em municípios próximos a outros com taxas baixas. Esse padrão pode ser decorrente da flutuação aleatória relacionada a peque- nas populações. As taxas variaram entre 0 a 36,35 casos por 100 mil habitantes, 0 a 40,82 casos por 100 mil habitantes e 0 a 41,57 casos por 100 mil habitantes para os períodos de 1988 a 1992, 1993 a 1997 e 1998 a 2002, respectivamente. O Município de Juiz de Fora, o segundo com maior registro de casos de HIV/AIDS no estado (o primeiro é a capital Belo Horizonte), apresentou taxas médias de incidência para os períodos de 1988 a 1992, 1993 a 1996 e 1997 a 2002, respectivamente, de 26,05, 40,82 e 41,57 por 100 mil habitantes.

Com o objetivo de visualizar as tendências globais de espalhamento da epidemia, empregou-se a suavização dos indicadores utilizandose o estimador bayesiano empírico local. A Figura 1 ilustra as taxas médias de incidência brutas e as taxas reestimadas pelo estimador bayesiano empírico local. A interiorização da epidemia pode ser visualizada ao longo dos períodos, e observa-se uma identificação menos fragmentada dos municípios com maior concentração de casos, incluindo o Município de Juiz de Fora e adjacências. As taxas de incidência estimadas pelo modelo bayesiano situaram-se entre 0 e 24,94 por 100 mil habitantes, 0 e 39,40 por 100 mil habitantes e 0 e 40,61 por 100 mil habitantes, para o primeiro, segundo e terceiro períodos, respectivamente.

As taxas médias de mortalidade por HIV/AIDS em maiores de 15 anos foram calculadas para os três períodos do estudo. Verificou-se um aumento da mortalidade por HIV/AIDS entre o primeiro e o segundo períodos, e um declínio no terceiro período, mas, devido à provável flutuação aleatória do indicador, ficou demonstrado um padrão irregular, tendo as taxa de mortalidade variado de 0 a 12,12 por 100 mil habitantes, entre 1988 e 1992, 0 a 28,92 por 100 mil habitantes, entre 1993 e 1996, e de 0 a 14,95 por 100 mil habitantes, entre 1997 a 2002. Juiz de Fora apresentou taxas de mortalidade de 5,08 por 100 mil habitantes, 19,35 por 100 mil e 14,95 por 100 mil para o primeiro, segundo e terceiro períodos, respectivamente.

As taxas médias de mortalidade foram reestimadas pelo estimador bayesiano Empírico e permitiram verificar o aumento da mortalidade por HIV/AIDS do primeiro para o segundo período, e o declínio no terceiro período, por meio de um padrão mais regular daquele apontado pelas taxas brutas. As taxas médias brutas e as taxas médias suavizadas estão ilustradas na Figura 2. As taxas de mortalidade reestimadas situaram-se entre 0 e 4,89 por 100 mil habitantes, 0 e 18,81 por 100 mil habitantes e 0 e 14,60 por 100 mil habitantes. O Município de Juiz de Fora e municípios adjacentes encontram-se como um foco de maior concentração de óbitos.

O fluxo intermunicipal de internação hospitalar construído para o ano de 1996 contabilizou 
Taxas médias de incidência de HIV/AIDS (por 100 mil habitantes) e taxas médias estimadas pelo método bayesiano empírico local, em maiores de 15 anos, por município de residência. Macrorregiões de Saúde Sudeste e Leste do Sul, Minas Gerais, Brasil, para os períodos 1988-1992, 1993-1996, 1997-2002.
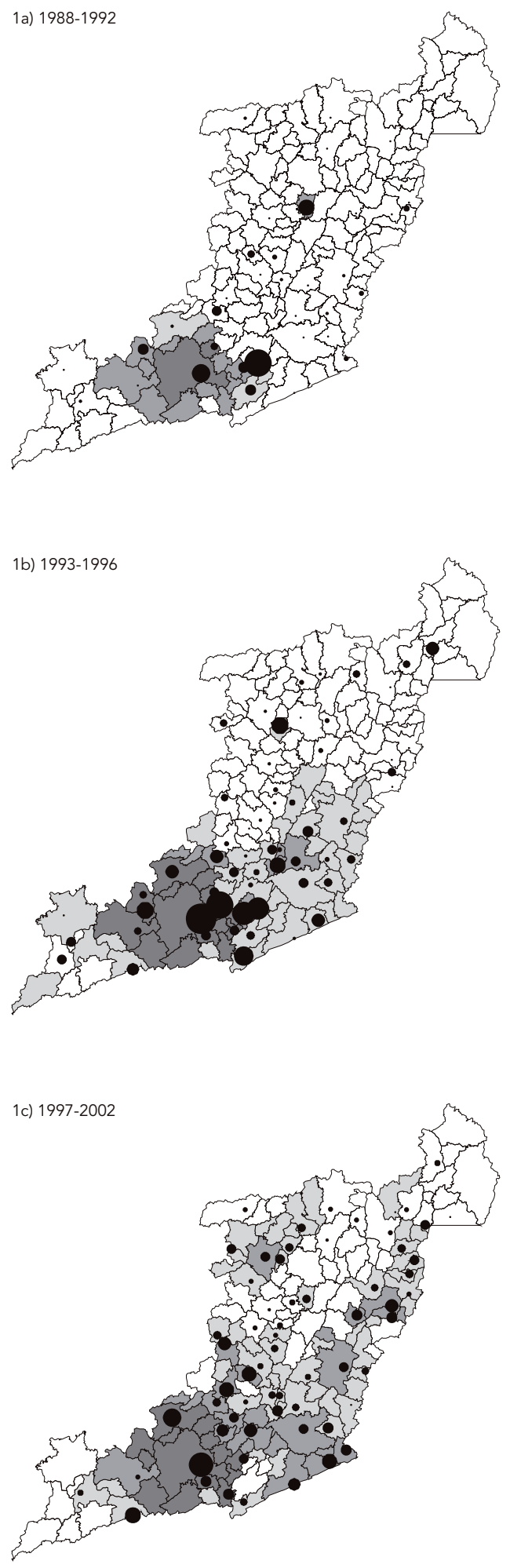

Taxa média de incidência

20

- 10

- 2

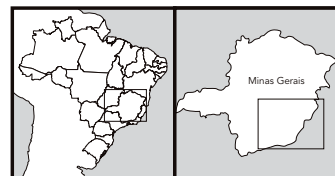

Taxa de incidência

estimador bayesiano empírico
20 a 40
10 a 20
5 a 10
0 a 5 
Figura 2

Taxas médias de mortalidade de HIV/AIDS (por 100 mil habitantes) e taxas médias estimadas pelo método bayesiano empírico local, em maiores de 15 anos, por município de residência. Macrorregiões de Saúde Sudeste e Leste do Sul, Minas Gerais, Brasil, para os períodos 1988-1992, 1993-1996, $1997-2002$.
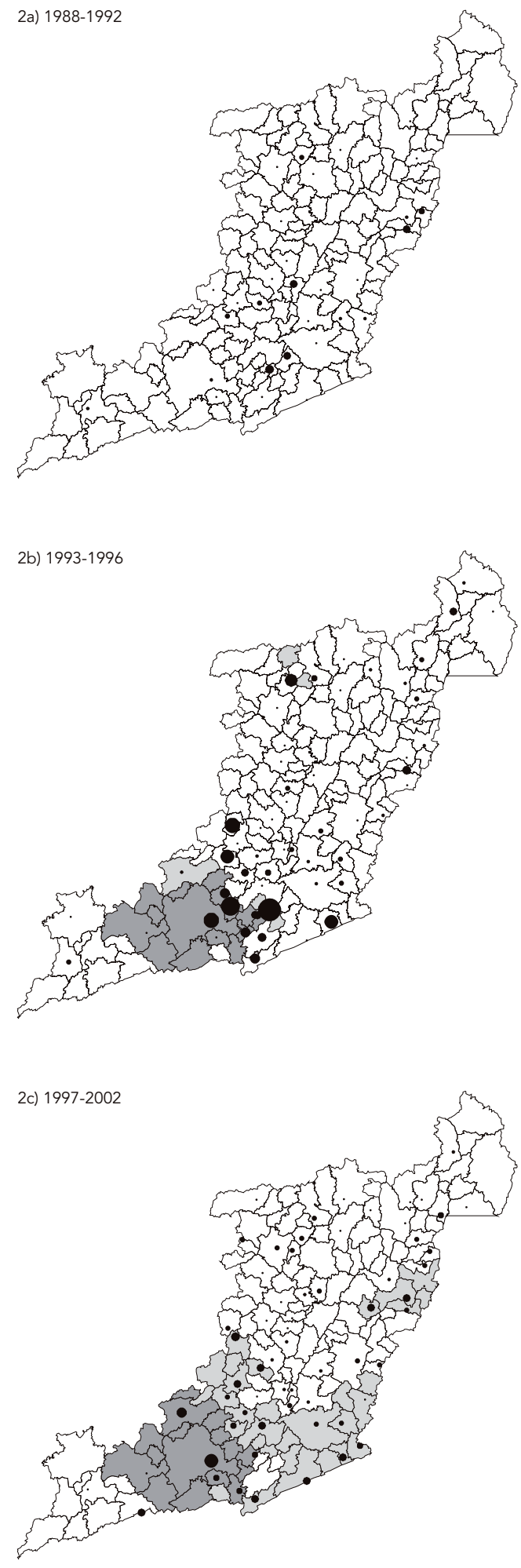

Taxa média de mortalidade

- 20

- 10

- 2
Taxa de mortalidade estimador bayesiano empírico

10 a 20

5 a 10

0 a 5 
151 internações por HIV/AIDS em maiores de 15 anos, de residentes nos municípios da Zona da Mata. Todas as internações ocorreram em Juiz de Fora, nas duas unidades hospitalares referenciadas pelo SUS para internação em HIV/AIDS. Do total de internações, 82,12\% são de residentes no próprio município, e o restante, de residentes nos demais municípios da mesorregião.

O fluxo construído para o ano 2004 contabilizou 852 internações hospitalares por HIV/AIDS. A maioria (96,83\%) ocorreu em hospitais de referência em Juiz de Fora. Do total de 825 internações efetuadas neste município, $68,85 \%$ foram de residentes do próprio município e $31,15 \%$ originaram de residentes dos demais municípios da Zona da Mata.

Desse modo, configurou-se um padrão de fluxos intermunicipais de internação hospitalar que em geral se mantém dentro dos limites da região. Das 852 internações ocorridas em 2004, de residentes nos municípios da mesorregião, 99,3\% ocorreram em municípios da Zona da Mata (Juiz de Fora, Muriaé, Ponte Nova, Andrelândia) e apenas $0,7 \%$ ocorreu em municípios não pertencentes a mesma (Belo Horizonte e Montes Claros).

O hospital cadastrado para atendimento em HIV/AIDS situado em Muriaé, contabilizou 18 internações em 2004. Dessas, 11 foram de residentes do próprio município, e 7 de residentes em municípios vizinhos. Todavia, 36 internações de residentes em Muriaé foram registradas em hospitais referenciados de Juiz de Fora, no mesmo ano.

\section{Discussão}

Os achados do presente estudo evidenciaram o processo de interiorização da epidemia de HIV/ AIDS na Zona da Mata de Minas Gerais, corroborando com estudos realizados em outras regiões do país 2,3,6.

O padrão de distribuição espacial de casos e óbitos por HIV/AIDS, nos municípios da Zona da Mata, mostrou um crescimento mais intenso da epidemia nos municípios da macrorregião de saúde Sudeste, onde se situa Juiz de Fora. Este município tem mantido, desde o início da epidemia, as maiores taxas de incidência e pode ter um papel de centro de difusão do HIV na região. Dentre as possibilidades de expansão de epidemias no espaço destacadas por Hagget 20 e reiteradas por Wallace \& Wallace 21, incluem-se a difusão por meio de contigüidade e a difusão hierárquica por redes. A difusão por meio de contigüidade ocorre pelo contato entre áreas adjacentes devido à freqüência de interações entre habitantes de áreas vizinhas, semelhante ao padrão sócio-espacial evidenciado neste estudo. A difusão por redes permite a transmissão dentro de comunidades, entre pessoas participantes de redes sociais, mesmo que distantes. Ambas pressupõem interação entre semelhantes, logo, permitem reproduzir a estrutura sócio-espacial da unidade de análise. Deve-se ressaltar, porém, que o processo de difusão não é homogêneo e produz uma mudança no perfil da epidemia, à medida que esta alcança novas populações 22. A centralidade de Juiz de Fora, sua importância social e econômica como pólo comercial, industrial, educacional e tecnológico da região, demanda intensa circulação de pessoas e movimentos migratórios. O padrão de distribuição espacial da AIDS evidenciado neste trabalho demonstra o importante papel das conexões estabelecidas entre cidades, por mais que distantes, num processo de difusão hierárquica, ao mesmo tempo em que se dá um espalhamento por contigüidade, atingindo municípios próximos a Juiz de Fora e outras cidades-pólo da região, como Muriaé, Cataguases, Leopoldina, Ubá e Manhuaçu. A atratividade dessas cidades é favorecida por um conjunto de redes que escoam produtos, direcionam os movimentos de migração e concentram informação e recursos.

A convergência de quase todo o fluxo intermunicipal de internação hospitalar de portadores de HIV/AIDS residentes na Zona da Mata para o Município de Juiz de Fora aponta para uma articulação bem regionalizada desse serviço na região, respeitando os limites preconizados pelo PDR. No entanto, comprova a pouca ou nenhuma utilização dos hospitais de outros municípios cadastrados como referência para internações de casos de HIV/AIDS. Segundo a Coordenação Estadual de DST/AIDS, o Município de Juiz de Fora participa com duas unidades hospitalares das cinco cadastradas para internações hospitalares 14 . Além de Juiz de Fora, os municípios de Leopoldina, Ponte Nova e Muriaé possuem, cada um, uma unidade hospitalar cadastrada, mas apenas a unidade de Muriaé participou com somente 2,11\% das internações ocorridas em 2004.

Tal resultado sugere as seguintes indagações: quais os motivos da internação de pacientes portadores de HIV/AIDS da Zona da Mata apenas nos hospitais cadastrados de Juiz de Fora e não nos dos municípios de menor porte? A internação de quase todos os pacientes em Juiz de Fora seria indicativa de precária qualidade na assistência oferecida nos outros hospitais cadastrados pela Coordenação Estadual de DST/AIDS nos municípios menores? Um aspecto a considerar é se a natureza complexa da atenção hospitalar aos pacientes de AIDS poderia explicar a convergência do fluxo intermunicipal de internação hospitalar 
para o Município de Juiz de Fora, sem a participação de outros hospitais cadastrados como referência pela Coordenação Estadual de DST/ AIDS. Mackee \& Healy (2000, apud Mendes 23, p. 42), afirmam que "o acesso à atenção hospitalar é menos suscetível à distância”, ao contrário dos serviços de atenção primária, preventiva e de urgência. E complementa que ganhos de escala e de escopo, além de aumentar a eficiência, são importantes para a melhoria da qualidade dos serviços. Para o autor, a diretriz de descentralização dos serviços de saúde apresenta fortalezas e debilidades. Se a municipalização das ações de saúde tem se mostrado adequada para a maior parte das ações básicas de saúde, os serviços que envolvem maior capacidade especializada e tecnológica têm sua eficiência e qualidade comprometidas com o processo de descentralização.

Entretanto, cabe ressaltar que o grau de eficiência dos tratamentos realizados "fora do domicílio" envolve realidades distintas, que variam fortemente entre os municípios exportadores de pacientes. Em estudo realizado para mapeamento das redes de atenção hospitalar do SUS, Oliveira et al. 24 observaram desigualdades regionais no acesso ao serviço de saúde, e como estabelecido amplamente na literatura, constataram que as pessoas percorriam distâncias maiores para obter assistência de serviços mais complexos.

A Zona da Mata de Minas Gerais, em quase sua totalidade é composta por pequenos municípios, que não possuem recursos técnicos e financeiros suficientes para aportar um serviço especializado em HIV/AIDS. Assim sendo, esses municípios referenciam seus pacientes particularmente para Juiz de Fora, onde seriam diagnosticados e tratados.

Como vimos, o problema da descentralização não diz respeito unicamente à internação hospitalar. $\mathrm{O}$ acesso a serviços de prevenção, aconselhamento, diagnóstico e tratamento com terapia anti-retroviral demanda atendimento ambulatorial especializado. Em razão da baixa qualidade dos dados ambulatoriais disponíveis não foi possível neste estudo avaliar o acesso do portador de HIV/AIDS a essas ações e serviços de saúde. De qualquer forma, pode ser comentado o fato de $o$ atendimento ambulatorial dispensador de tratamento anti-retroviral para toda a Zona da Mata ser inteiramente centralizado em Juiz de Fora. Não seria prioritária a descentralização microrregional desse tipo de serviço, que demanda menos tecnologia comparativamente à assistência hospitalar?

O serviço de atendimento especializado, estrutura ambulatorial dispensadora de anti-retrovirais, apresenta uma estrutura multidisciplinar, composta por médico clínico geral (ou infectologista), enfermeiro, assistente social, psicólogo e farmacêutico. Esse serviço não demanda alta tecnologia, visto que o apoio laboratorial pode se manter centralizado no nível da macrorregião. Municípios-pólo de microrregiões como Viçosa, Ponte Nova, Ubá, Muriaé, Leopoldina e Cataguases poderiam ser avaliados em relação à possibilidade de aportarem essa modalidade de atenção.

A análise da distribuição espacial da ocorrência de casos e óbitos foi melhor efetuada a partir da suavização das taxas médias de incidência e de mortalidade, por intermédio da utilização do método bayesiano empírico local quando comparadas às taxas médias de incidência e mortalidade brutas.

A integração dos programas TABWIN e R e sua interface com o TerraView, todos programas de distribuição gratuita utilizados neste estudo, permitiram ampliar as análises dos dados fornecidos pelo Ministério da Saúde, por meio do DATASUS, constituindo uma ferramenta eficaz para os pesquisadores e, em especial, para os profissionais das diversas esferas do SUS.

Um dos principais limites deste estudo ecológico foi a indisponibilidade de informações acerca do atendimento ambulatorial dos portadores de HIV/AIDS. Essas informações expressariam as condições do paciente por ocasião do diagnóstico e sua evolução terapêutica e permitiriam a realização de estudos comparativos de avaliação dos serviços, contrastando a assistência recebida por residentes nos municípios de maior porte com estrutura especializada, com aquela recebida pelos pacientes residentes nos pequenos municípios. Estudos analíticos, longitudinais e de sobrevida auxiliariam na compreensão desses processos. 


\section{Resumo}

O objetivo deste artigo foi analisar o processo de interiorização da epidemia de AIDS e investigar o acesso aos serviços em HIVIAIDS, entre 1988-2002, na Zona da Mata, Minas Gerais, Brasil. Realizou-se estudo ecológico, com abordagem têmporo-espacial. Construiuse um fluxo de internação hospitalar, tendo como referência pacientes maiores de 15 anos, internados pelo Sistema Único de Saúde, nos anos de 1996 e 2004, residentes nos municípios da região. Foram registrados 2.469 casos de AIDS em maiores de 15 anos no período; as taxas médias de incidência e mortalidade foram calculadas e reestimadas pelo estimador bayesiano empírico local para representar de maneira mais clara os municípios com maior concentração de casos e de óbitos. Verificou-se a interiorização da epidemia. Juiz de Fora é o município com maior número de casos e provável centro difusor da AIDS na região. A assistência hospitalar dos casos de AIDS da Zona da Mata está concentrada nesse município. Destaca-se necessidade de investigações adicionais sobre causas da não-participação de hospitais de referência em outros municípios da região na gerência e prestação de cuidados aos pacientes que vivem com HIVIAIDS.

HIV; Síndrome de Imunodeficiência Adquirida; Hospitalização; Análise Espacial

\section{Colaboradores}

C. T. Reis concebeu e planejou o estudo, levantou os dados, analisou e interpretou os resultados e redigiu o texto. D. Czeresnia revisou o texto, contribuiu no planejamento do estudo, na análise e interpretação dos resultados. C. Barcellos contribuiu no planejamento do estudo, na análise e interpretação dos resultados. W. S. Tassinari colaborou na execução da construção dos mapas e análise dos dados.

\section{Agradecimentos}

Apoio financeiro: Conselho Nacional de Desenvolvimento Científico e Tecnológico mediante bolsa concedida durante a realização de mestrado acadêmico, na Escola Nacional de Saúde Pública Sergio Arouca, Fundação Oswaldo Cruz, cujo trabalho de pesquisa originou este artigo.

\section{Referências}

1. Szwarcwald CL, Bastos FI, Barcellos C, Esteves MA, Castilho EA. Dinâmica da epidemia de AIDS no Município do Rio de Janeiro, no período de 198896: uma aplicação de análise estatística espaçotemporal. Cad Saúde Pública 2001; 17:1123-40.

2. Szwarcwald CL, Bastos FI, Esteves MA, Andrade CLT. A disseminação da epidemia de AIDS no Brasil, no período de 1987-1996: uma análise espacial. Cad Saúde Pública 2000; 16 Suppl 1:S7-19.

3. Brito AM, Castilho EA, Szwarcwald CL. AIDS e infecção pelo HIV no Brasil: uma epidemia multifacetada. Rev Soc Bras Med Trop 2000; 34:207-17.

4. Fonseca MGP, Travassos C, Bastos FI, Silva NV, Szwarcwald CL. Distribuição social da AIDS no Brasil, segundo participação no mercado de trabalho, ocupação e status sócio-econômico dos casos de 1987 a 1998. Cad Saúde Pública 2003; 19:1351-63.

5. Parker R, Camargo Jr. KR. Pobreza e HIV/AIDS: aspectos antropológicos e sociológicos. Cad Saúde Pública 2000; 16:89-102.
6. Bastos FI, Barcellos C. Geografia social da AIDS no Brasil. Rev Saúde Pública 1995; 29:52-62.

7. Rodrigues-Júnior. AL, Castilho EA. A epidemia de AIDS no Brasil, 1991-2000: descrição espaço-temporal. Rev Soc Bras Med Trop 2004; 37:312-7.

8. Ministério da Saúde. Boletim Epidemiológico AIDS 2003; Ano XVI, no 1.

9. Coordenadoria de DST/AIDS, Secretaria de Estado da Saúde de Minas Gerais. AIDS em Minas Gerais - 1982-2004. http://www.saude.mg.gov.br:8180/ saude/viewController.jsp?page $=57$ (acessado em 24/Out/2004).

10. Proietti FA, Caiaffa WT. Minas Gerais. In: Ministério da Saúde, organizador. Simpósio satélite: a epidemia de AIDS no Brasil - situação e tendências. Brasília: Ministério da Saúde; 1997. p. 193-216.

11. Marins JRP, Jamal LF, Chen SY, Barros MBA, Hudes ES, Barbosa AA, et al. Dramatic improvement in survival among adult Brazilian AIDS patients. AIDS 2003; 17:1675-82. 
12. Nemes MIB, Castanheira ERLC, Melchior R, Alves MTSB, Basso CR. Avaliação da qualidade da assistência no programa de AIDS: questões para a investigação em serviços de saúde no Brasil. Cad Saúde Pública 2004; 20:310-21.

13. Secretaria de Estado da Saúde de Minas Gerais. Plano Diretor de Regionalização PDR. http://www. saude.mg.gov.br:8180/saude/viewController. jsp?page $=10$ (acessado em 03/Jan/2005)

14. Coordenadoria de DST/AIDS, Secretaria de Estado da Saúde de Minas Gerais. Hospitais que atendem pacientes com HIV/AIDS. http://www. saude.mg.gov.br:8180/saude/viewController. jsp?page $=57$ (acessado em 24/Out/2004).

15. Barbosa MTS, Struchiner C J. Estimativas no número de casos de AIDS: comparação de métodos que corrigem o atraso de notificação. In: Ministério da Saúde, organizador. Simpósio satélite: a epidemia de AIDS no Brasil - situação e tendências. Brasília: Ministério da Saúde; 1997. p. 15-26.

16. Assunção RM. Estatística espacial com aplicações em epidemiologia, economia, sociologia. São Carlos: Associação Brasileira de Estatística; 2001.

17. Geltman A. Bayesian data analysis. London/New York: Chapman \& Hall; 1995.

18. Organização Mundial da Saúde. Manual da classificação estatística internacional de doenças, lesões e causas de óbitos - nona conferência de revisão. São Paulo: Centro Brasileiro de Classificação de Doenças em Português; 1985.
19. Organização Mundial da Saúde. Classificação estatística internacional de doenças e problemas relacionados à saúde, 10a Revisão. v. 1. São Paulo: Centro Colaborador da OMS para a Classificação de Doenças em Português; 1995.

20. Hagget $P$. The geographical structure of epidemics. Oxford: Oxford University Press; 2000.

21. Wallace R, Wallace D. Socioeconomic determinants of health: community marginalisation and the diffusion of disease and disorder in the United States. BMJ 1997; 314:1341-5.

22. Tomazelli J, Czeresnia D, Barcellos C. Distribuição dos casos de AIDS em mulheres no Rio de Janeiro: uma análise espacial. Cad Saúde Pública 2004; 19:1049-61.

23. Mendes EV. Os grandes dilemas do SUS. Tomo II. Salvador: Casa da Qualidade Editora; 2001.

24. Oliveira EXG, Carvalho MS, Travassos C. Territórios do Sistema Único de Saúde - mapeamento das redes de atenção hospitalar. Cad Saúde Pública 2004; 20:386-402.

Recebido em 20/Dez/2006

Versão final reapresentada em 04/Set/2007

Aprovado em 08/Out/2007 